\title{
"LA AGARRADURA ME LA ATIENDO EN LA IGLESIA": EL DIABLO COMO SÍMBOLO HEGEMÓNICO EN EL PLURALISMO MÉDICO AYMARA PENTECOSTAL
}

\author{
"I HEAL MY AGARRADURA AT CHURCH": \\ THE DEVIL AS HEGEMONIC SYMBOL IN THE AYMARA-PENTECOSTAL \\ MEDICAL PLURALISM
}

\author{
Carlos Piñones Rivera ${ }^{1}$, Miguel Ángel Mansilla Agüero ${ }^{1}$ y Wilson Muñoz Henríquez ${ }^{2,3}$
}

\begin{abstract}
Existen pocos estudios acerca del pluralismo médico entre los aymaras de Chile. El presente artículo presenta y analiza material de campo producto de una etnografía respecto de la articulación de saberes médicos realizada el 2011-2012 entre los agricultores de Camiña (Región de Tarapacá, Chile), focalizándose la díada de los Saberes Médicos Andino y Pentecostal. El análisis problematiza la dificultad teórica de concebir la operatividad resultante de las articulaciones de saberes médicos, desarrolla la utilidad que el concepto de símbolo hegemónico (Ernesto De Martino) puede tener para superar esta aporía y explora la hipótesis de que el diablo podría constituir dicho símbolo hegemónico para el caso estudiado, iluminando su función al interior de la producción de relaciones de hegemonía/subalternidad entre los Saberes Médicos Andino y Pentecostal.
\end{abstract}

Palabras claves: salud pueblos indígenas, salud intercultural, antropología médica crítica, etnografía, Tarapacá.

There are few studies on medical pluralism among the Aymaras of Chile. This article presents and analyzes field material of an ethnography on the articulation of medical knowledge made between the years 2011-2012 with farmers from Camiña (TarapacáChile), focusing on the dyad of Andean and Pentecostal Medical Knowledge. The analysis problematizes the theoretical difficulty of conceiving the operativeness resulting from the articulation of medical knowledge, develops the usefulness that the concept of hegemonic symbol (Ernesto De Martino) may have in overcoming this aporia and explores the hypothesis that the devil could be seen as the hegemonic symbol for the case studied, illuminating its role within the generation of hegemonic/subalternate relationships between Andean and Pentecostal medical knowledge.

Key words: Indigenous people's health, intercultural health, critical medical anthropology, ethnography, Tarapacá.

Si bien el campo de estudios del pluralismo médico es vasto y de larga data (Cant y Sharma 1999; Leslie 1980; Menéndez 2003; PerdigueroGil 2006; Ramírez Hita 2005; Stoner 1986), muy pocas investigaciones han abordado su presencia entre los aymaras en Chile. Esto se debe, entre otras razones, a que estos estudios sobre el Proceso Salud/Enfermedad/Atención ${ }^{1}$ se han centrado en los problemas de salud adoptando preferencialmente una mirada biomédica (Caba et al. 2011; Carrasco et al. 2012; Lagos et al. 2011; Makela et al. 1978; Rothhammer et al. 2005) o la del Saber Médico Andino (Cuadro 2008; Gavilán et al. 2009; 2011; Grebe 1983; Van Kessel 1983, 1985).
En general estas investigaciones han considerado a los saberes médicos de forma aislada, no abordando sus procesos de articulación. Una excepción son los trabajos de Van Kessel y Guerrero (1987) y Guerrero (1995) sobre las articulaciones de los saberes médicos andino y pentecostal, siendo pioneros en este campo en Chile. Pero si bien sus aportes son significativos a nivel del material empírico aportado y el análisis conceptual, poseen una limitación parcial: no asumen la totalidad de los saberes que conforman el pluralismo médico ${ }^{2}$, siendo imposible conocer sus articulaciones internas.

En este artículo analizaremos este fenómeno utilizando una mirada relacional, que nos permitirá

\footnotetext{
1 Instituto de Estudios Internacionales (INTE), Universidad Arturo Prat. Av. Prat \# 2120, Iquique, Chile. carlospinonesrivera@gmail.com; mansilla.miguel@gmail.com

2 Facultad de Educación y Humanidades, Universidad de Tarapacá. Av. 18 de septiembre \# 2222, Arica, Chile. wilsonsocio@gmail.com

3 Laboratoire d'Anthropologie Sociale (LAS), École des Hautes Études en Sciences Sociales (EHESS). 52 rue du CardinalLemoine, 75005 Paris, Francia.
} 
ampliar la caracterización de los problemas de salud. A partir de la consideración del Proceso Salud/ Enfermedad/Atención como una manifestación del pluralismo médico, nos interesa explorar algunas de las articulaciones más significativas que emergen entre los saberes médicos de los agricultores precordilleranos aymaras de Camiña (Región de Tarapacá, Chile). En concreto, analizaremos las relaciones entre el Saber Médico Andino y el Saber Médico Pentecostal ${ }^{3}$. Para esto expondremos un fragmento del material de campo que evidencia la emergencia de estas relaciones y luego analizaremos la utilidad del concepto símbolo hegemónico (De Martino 1999) para comprender las nuevas formas de eficacia producidas por dichos procesos de articulación (Menéndez 1994). Así, esperamos iluminar las diversas determinaciones socioculturales y económico-políticas que inciden en el Proceso Salud/Enfermedad/Atención, como también la forma en que esta interrelación alimenta dichas determinaciones, contribuyendo a restituir la complejidad del proceso (Crandon-Malamud 1991; Menéndez 2003; Ramírez Hita 2005).

\section{Materiales y Métodos}

El material de campo en el cual se basa nuestro análisis es fruto del trabajo etnográfico realizado con los agricultores aymaras de Camiña en la Región de Tarapacá (Chile) entre 2011 y 2012. Estos datos forman parte de una investigación mayor, cuyo objetivo fue describir y analizar los saberes médicos y sus procesos de articulación y configuración de relaciones de hegemonía/subalternidad (Piñones Rivera 2015).

El diseño metodológico se estructuró específicamente para conocer cada uno de estos saberes médicos. Primero, utilizamos la observación participante como técnica principal de recolección de información. Para obtener información sobre el Saber Andino se realizaron observaciones en el centro de atención "Casa del Kollire" (co-administrado por la Asociación Kollire y la Municipalidad), así como también en los hogares de algunos qullirinaja, yatirinaja ${ }^{4}$ o pacientes donde se realizaban las atenciones. Para el caso del Saber Pentecostal se asistió a las sesiones de culto y a las concentraciones pentecostales realizadas en la iglesia local, y se visitaron las casas de personas enfermas para observar las vigilias. En el caso del Saber Biomédico las observaciones se llevaron a cabo en la posta de atención rural. Finalmente, para conocer el Saber de Autoatención se observó la convivencia cotidiana en los espacios domésticos. Los tópicos claves abordados fueron las prácticas de atención a los problemas de salud.

De manera complementaria se realizaron 68 entrevistas a representantes de los distintos saberes médicos ${ }^{5}$. Fueron entrevistados 10 representantes del Saber Andino, 13 del Pentecostal, seis del Biomédico, 28 del de Autoatención y 10 correspondientes a la categoría "Otros informantes". Mientras que 15 personas declararon poseer una adscripción religiosa pentecostal, ocho adventistas, 47 católicas, una persona se definió como atea y una señaló no tener adscripción religiosa. De los entrevistados, 35 corresponden a mujeres y 37 a hombres. De este total solo dos tenían menos de 20 años, 16 entre 20 y 40 años, 23 entre 40 y 60 años y 31 más de 60 años. Los tópicos centrales de las entrevistas fueron las diversas enfermedades sufridas y conocidas, poniendo especial atención en sus procesos de desarrollo, causas y prácticas de atención.

De esta manera, mientras de la observación participante obteníamos información directa sobre las prácticas de atención correspondientes a cada saber, las entrevistas nos entregaban información de las representaciones asociadas. Esto nos permitió generar una interpretación de los elementos ideológicos característicos de los saberes médicos, así como de los procesos de articulaciones que se dan entre ellos.

La información fue sometida a un análisis comparativo inicial de las distintas características ideológicas de los saberes médicos. Luego se identificaron los fenómenos o procesos donde se evidenciaba la presencia de elementos del Saber Andino y del Saber Pentecostal operando en las representaciones y prácticas de los especialistas de los distintos saberes en cuestión. De este conjunto se seleccionaron aquellos que destacaban por su recurrencia, interés teórico o grado de significatividad en el Proceso Salud/Enfermedad/Atención local. Posteriormente se verificó que los fenómenos y procesos relatados no fueran excepcionales, sino que formaran parte de las prácticas cotidianas y el sentido común de los especialistas o legos. Por último, se analizaron los rasgos ideológicos que obstaculizaban y facilitaban las articulaciones entre los saberes médicos concretos, así como la contribución que estas articulaciones generaban en el posicionamiento hegemónico de un determinado 
saber. El análisis en general se realizó siguiendo los principios teórico-metodológicos de la antropología médica crítica, lo que nos permitió centrarnos en las articulaciones y transacciones entre saberes hegemónicos y subalternos ${ }^{6}$. En conjunto, las distintas etapas del análisis antes mencionadas y la permanente reflexión sobre los múltiples niveles involucrados, permitieron la necesaria triangulación metodológica.

\section{Resultados y Discusión}

\section{El campo articulatorio: la atención de los problemas del saber andino en la iglesia evangélica pentecostal (IEP)}

Uno de los datos más significativos de nuestro trabajo etnográfico, y que llama especialmente la atención por su ausencia en la bibliografía sobre los aymaras en Chile, es que muchos de los problemas de salud tradicionalmente tratados por los yatirinaja son atendidos actualmente por el Saber Pentecostal ${ }^{7}$. Como veremos a lo largo del artículo, uno de los ejemplos más significativos al respecto es la "agarradura". En lo que sigue, nos centraremos en las actuales prácticas de protección y terapéuticas que utiliza la población aymara pentecostal frente a las "agarraduras", y evidenciaremos algunos de los procesos articulatorios más significativos de este fenómeno.

\section{Prácticas de protección}

El espacio aymara está conformado por entidades territoriales específicas. Cotidianamente la comunidad convive y establece relaciones de reciprocidad con mallkus, t'allas, serenos, chullpas, condenados, etc. ${ }^{8}$ Esto configura una topología específica ${ }^{9}$ que distingue en el espacio lugares "fuertes", "bravos", "fieros" o "malos parajes", en los cuales es peligroso transitar, por lo que se les debe guardar un respeto especial, manifiesto en prácticas como las ch'allas o phawas ${ }^{10}$. Dichas entidades territoriales participan del Proceso Salud/Enfermedad/Atención, ya que algunas de las principales enfermedades que sufren los camiñanos corresponden a "agarraduras" $(\text { katjata })^{11}$, es decir, enfermedades que se producen por la captura ("agarre") del ánimo por parte de las entidades territoriales ${ }^{12}$.

En la literatura especializada la "agarradura" ha sido entendida la mayoría de las veces como una enfermedad espiritual (Ramírez Hita 2005), donde existe la creencia de que un espíritu se apodera del alma de la persona, produciendo una sintomatología que puede conducir a la muerte. Sus causas son asociadas principalmente a la "debilidad del ánimo" y a la intervención de diversos seres maléficos o demonios (Gavilán et al. 2009).

En nuestra experiencia de campo, sin embargo, cuando las personas explicaban la causa de la enfermedad que padecían, señalaban que esta habría ocurrido al pasar por un "mal paraje" y en una "mala hora", cuestión que supone y devela una lógica etiológica particular. Esto es central, pues la "agarradura", antes que especificarse por sus síntomas, lo hace por el conocimiento de sus causas, las que pasan por reconocer a la entidad territorial y las circunstancias que acompañaron al infortunio en sus orígenes. Cuestión que se evidencia en las palabras de los nativos, quienes indefectiblemente las localizan (agarraduras de cerro, de vertiente, de pachamama, etc.), y buscan sus causas interrogando a la hoja de coca por parte del yatiri, o empleando el naipe, el cual es consultado usualmente para conocer los sucesos que acompañaron el momento de la "mala hora".

Ante la posibilidad de enfermar debido a la relación que los aymaras establecen con las entidades territoriales, el Saber Andino ha instituido múltiples prácticas precautorias basadas en el cuidado de las relaciones de reciprocidad ${ }^{13}$. Por ello se cuidan de manifestar el respeto por aquellos lugares, a través de prácticas como "hacer las veces" (ch'allar) con alcohol, evitar caminar por estos lugares para no perturbar su calma, entre otras.

En el escenario cultural aymara, uno de los procesos articulatorios que registramos se configuró con la expansión de la religión pentecostal en la región. Entre los diversos efectos que este proceso generó, se destaca la resignificación específica del espacio: en concreto, los "malos parajes" se transformaron en lugares donde se aparece el "maligno" (Satanás). Dicha resignificación transformó también las prácticas de protección utilizadas tradicionalmente en las relaciones con las entidades territoriales, pues desde la óptica pentecostal, todas las formas de reciprocidad con estas entidades son connotadas como una forma de "ponerse a los pies del maligno". Por esta razón, la comunidad desarrolló una serie de prácticas que buscan protegerla de su influjo, como se evidencia en el siguiente testimonio. 


\section{C: ¿Cómo se protegen del diablo?}

V.F.: Nosotros le oramos no más al señor.

Nuestra religión nos enseña a clamar: nosotros decimos 'la sangre de Cristo tiene poder'; cuando tenemos encima un miedo reprendemos. Entonces cuando tenemos miedo, ya se va, se va el mal, así nosotros le llamamos reprender.

Como un mes atrás yo sentí un ruido fuerte: 'muuu' hace... 'muuu'. El cerro se mueve... mi mami lo ha visto muchas veces. Hace un mes atrás Don J. lo vio... dice que se movía y él, como va a la iglesia, lo reprendió y se quedó quietecito.

C: Pero ¿por qué se mueve?

V.F: El diablo lo mueve poh... ningún cerro tiene vida. Está apoderado ahí porque hay mucha riqueza

Una situación similar ocurre con las vertientes. Según la topología andina, en las vertientes habita el sireno. Sin embargo, el pentecostalismo no posee una relación de respeto con él, pues sería una manifestación más del maligno, debiendo ser ahuyentado a través de alabanzas y oraciones:

V.F.: Mi mamá me decía: 'mira está la sirena'. Me agarraba de la espalda mi mami, 'pero no tenga miedo', me decía, 'está el señor con nosotros y nos protege'. [...] 'si tu tenís miedo va a venir acá y no vai a poder dormir', me decía. 'Te va a llevar el alma', me decía... no hay que tener miedo. Mi mamá cantaba alabanza del señor de la iglesia, empezaba a cantar y cuando cantamos así coritos de Dios como que desaparecía... pero a mí me da miedo antes... era así como un vacío negro de lava, porque mi mamá dice que era profundo... mi mamá no me dejaba ir.

Es interesante apreciar que si bien la relación con los "lugares fuertes" generalmente implicaba un riesgo para la comunidad, pues la realización de los rituales suponía la posibilidad de un fracaso (por fallo de la memoria sobre la costumbre o porque dicha realización confluye justo con una "mala hora", etc.), normalmente las relaciones de reciprocidad estaban teñidas de una confianza en los resultados beneficiosos de estas relaciones. Con la resignificación que realizan los pentecostales de los "lugares fuertes", concebidos ahora como demoníacos, el espacio se transforma en una entidad totalmente amenazante: el diablo abstracto adquiere el rostro de los chullpas, vertientes, cerros, etc., mientras que el respeto que previamente recibían se trasmuta en temor al maligno ${ }^{14}$.

\section{Prácticas de curación}

Un segundo tipo de fenómeno que nos interesa tematizar son las prácticas de curación. Cuando las protecciones no bastan y las personas caen víctimas del maligno, ocurre una "agarradura". Pero la "agarradura" característica del Saber Andino es resignificada por el pentecostalismo, modificando el significado de sus causas, su proceso patogénico y su tratamiento. Un caso interesante de analizar son las "agarraduras de tierra", es decir, la agarradura que corresponde a la intervención de la pachamama. Un testimonio nos ayudará a clarificar esta idea:

C: Don Ch.M. Y si la persona acá, yo he escuchado que la tierra le agarra

Ch.M.: Claro, en la tierra está también el mal... en la tierra, también te toma, cuando tú tienes una debilidad espiritual la tierra te toma.

C: ¿Ese también Ud. lo puede tratar?

Ch.M.: Sí, también eso. Con solo, mayormente mucho se ocupa el salmo. Salmo es un rezo, entonces ud. le aplica al hombre o a la mujer con un salmo y el enemigo, que no se ve, se manda a cambiar el enemigo (...)

C: Oiga Don Ch.M. ¿eso que Ud. me explicaba de la agarradura, ud. la saca con el salmo?

Ch.M.: Salmo 91 es especial pa' eso. Con el salmo hay que ungir. La biblia dice hay que ungir con el aceite, con la autorización del padre celestial, todo con el salmo. Entonces ese mal se le corre, se le parte. Entonces una o dos veces hay que hacerle $\mathrm{y}$ ¡listo! con eso y se le quita.

C: ¿Y antes cuando Ud. era católico, Ud. como le hacía pa' sacar la agarradura?

Ch.M.: ¿Antes qué hacíamos? Estaba la qolla, el copal, [...] y se traía un animalito, [...] y con ese cuestioncita preparaba con qolla, untu. Qolla es una hierba, un monte; el unto es la grasa del animal; y después el 
copal que viene de Bolivia uno plomito; y el millo, porque el millo es sal de los malos, entonces con millo con todo hay que dar vuelta, que se llama antiguamente turca. C: ¿Daban vuelta el animal?

Ch.M.: Con animal daban vuelta al enfermo, salga todos los malos, y salía el malo apegaíto a ese animal. Se hace un cambio del humano a ese animal, entonces Ud. lo saca y lo carnea y toda la especie se quema y el enfermo se sanaba. Ése hacía antiguamente, un buen tiempo, alrededor de los 15-16 años más o menos, y ahora nada poh, no ocupábamos nada, solo salmo y la oración y se despeja.

Este tipo de experiencias articulatorias no solo poseen un alto valor etnográfico, sino también un gran potencial teórico, pues muestran cómo la IEP ha capitalizado elementos pertenecientes al Saber Andino, transformando las prácticas correspondientes a una de sus categorías diagnósticas, y fortaleciendo la resignificación demoníaca de la pachamama, entidad fundamental en la concepción andina.

La preparación de la ofrenda a la pachamama, que antes implicaba una cuidadosa composición de elementos acordes con su gusto, desaparece en su acepción positiva y es vista como un ritual más de la idolatría demoníaca. Así, la "agarradura de pachamama" debe ser tratada expulsando al demonio del cuerpo, a través de la utilización de un salmo protector. Por otro lado, desde la resignificación pentecostal del proceso de atención, el interlocutor deja de ser la entidad territorial y en su lugar emerge el dios cristiano, a quien se le solicita la autorización para que el salmo sea efectivo. De esta forma las entidades territoriales son excluidas de las relaciones de reciprocidad, transformándose en meros objetos de la animadversión y el repudio pentecostal.

Una configuración similar ocurre en la relación con los denominados gentiles ${ }^{15}$, como veremos en el siguiente relato de Don V.F.

V.F.: Yo casi como 18 años caminé por los caminos del señor... Pero ¿por qué yo me entregué? [...] cuando yo me junté el año 71 bajé a la costa con ganado, entonces siempre el animal se iba más arriba de aquí [...] más a la pampa había un corral grande y me puse a pirqarlos ${ }^{16}$ yo, el corral. Aquellos tiempos, sería de gentilares pues, los corrales... entonces yo, pirqamos, mi señora y mi hermana, pirqamos juntos... Así tiempo estuvo las piedras, como estuviera esta piedra así rodeado [...] Y ¡ya! Eché a los llamos al corral ya en la tarde, ya le sentí el dolor del estómago, como sonajera, [...] ¡chuuuta!, ya como que molesté al chullpa... el chullpa se me entró en la muela, el estómago, oh chullpa... ¡ya! Mientras yo estoy tomando el remedio así, iya listo!, si voy ahí no más.

Después que me fui a cordillera, me pescó en cordillera. Estaba en una siembra y ¡listo! no me recuerdo lo que es nada... ¡y me pescó firme, firme! [...] pero como ese día me dormí, al otro día me desperté, me habían hecho la turca ${ }^{17}[\ldots]$ Entonces me desperté y me vine pa' arriba ahí me vine. Años sufrí con eso oiga... enflaquecí... era más macizo.

C: ¿Y su estómago le seguía sonando? V.F.: O sea... que hace bulla. En amanecía hace bulla. En la tarde se inflama el estómago. Entonces ya estamos pagando a la gente. Mi hijo mayor también se enfermó, [...] no tenía plata pa' pagar poh [...] Entonces en una ya... me dice mi primo que viene el pastor. Tú que estai enfermo mejor entrégate al señor. Yo le dije ¡ya poh!, pero no cobra ni un cinco.

Yo acepté altiro [de inmediato] porque no tenía plata ni nada. Yo dije 'mejor voy a entregarme a mi camino' [...] Ya entregados dije yo, estaba de acuerdo, total que estamos caminando... a la final se enfermó ella... ¡ah, como que se empezó a enfermar toda la familia! Es que el diablo estaba trabajando ya.

C: ¿Y el Yatiri no le supo sanar bien?

V.F.: Supo sanarme a mí no más, pero el diablo pescó otro y otro... entonces ya estoy caminando, nos arrepentimos ya, pero otra vez seguí en el camino, ya pasé probando, a la final ya pasé en plena ${ }^{18}$, porque el grado está subiendo. Y cuando ya pasé en plena, me presenté allá [...]

[V.F.: Personificando al chullpa]: 'Tú me estás cuidando mi casa'

V.F.: Yo le estaba cuidando su casa de chullpa, por eso ahí está permaneciendo... está como en su casa el chullpa. Y así de 
tanto como su casa llegaba a pensar, ya en uno en uno estuve viendo la llijlla ${ }^{19}$, en uno ya llegué a pensar, me vino en la mente de afrontrarme con el chullpa.

[...] Entonces me vino a la mente voy a poner posición de la mano ${ }^{20}[\ldots]$

V.F.: [...] Y después al otro día me desperté y ya no había nada... y como que asusté parece... cómo era la cosa...

C: ¿Y su enfermedad se sanó?, ¿ahí mismo se sanó?

V.F.: Ha ido calmando, calmando hasta hoy día

C: ¿En visiones le pasó eso?

V.F.: Claro... ¡no si es cosa fuerte el chullpa!, ¡cosa seria!

En este dramático y emotivo relato, podemos apreciar cómo se articula el imaginario de los chullpas, propio del Saber Andino, con una forma de tratamiento pentecostal. La imposición de manos que opera en el plano espiritual y por medio de una visión espiritual, es lo que permite a la persona sanarse de los chullpas. El caso es esclarecedor sobre cómo, una vez que una persona ha asumido una estructura de significación del Proceso Salud/ Enfermedad/Atención cuyo eje es su relación con el dios pentecostal, su repertorio de prácticas y sentidos se modifica ${ }^{21}$. En este ejemplo, la relación con el chullpa está organizada desde un esquema bélico, en el cual la imposición de manos es un elemento fundamental, en tanto pone a disposición del fiel el poder de dios (Mansilla 2009).

¿Cómo entender estos procesos de articulación? Estas prácticas no pueden ser concebidas solamente desde el Saber Pentecostal, pues la imposición de manos evangélica que ahuyenta la presencia del maligno, opera y se muestra eficaz sobre el imaginario etiológico y patogénico de los chullpas. La constatación de esta articulación propone un desafío analítico relevante, ya que tendemos a pensar que si el Saber Pentecostal fue eficaz, es porque se dio una transformación completa o integral de sus (nuevos) creyentes, o una absorción de los elementos del Saber Andino en una lógica terapéutica diversa. La estrategia teórica clásica consiste en reducir esta relación a la operación de uno de sus elementos, utilizando para ello conceptos como adopción, asimilación o traducción (Menéndez 2003).

Sin embargo, esto no coincide con nuestra evidencia etnográfica. No encontramos saberes puros con lógicas totalizantes ni unitarias, sino saberes articulados, con elementos heterogéneos y contradictorios, cuyas lógicas fragmentarias operaban en lo concreto como unidad de lo diverso. En el testimonio antes mostrado, la lucha con el maligno pentecostal tiene un sustrato vivido que es el de la experiencia de los gentilares y este solo halla su sentido en el saber incorporado andino. Igualmente, solo alguien que ha sido suficientemente formado en el pentecostalismo puede experimentar las visiones espirituales y aplicar con eficacia la imposición de manos a los chullpas.

\section{La coexistencia coordinada y operativa de los saberes y el diablo como símbolo hegemónico}

En los ejemplos presentados no encontramos solo la coexistencia de elementos de ambos saberes (pentecostal y aymara), sino que dicha coexistencia es también operativa. De aquí surge un segundo desafío consistente en mostrar cómo los procesos articulatorios devienen operativos. La dificultad que esto supone es que tanto el sentido común del lego como el de los especialistas, explica el fenómeno de la eficacia invocando la coherencia, consistencia y sistematicidad de los saberes en cuestión ${ }^{22}$. De esta forma, una explicación desde las articulaciones aparece como una contradicción en los términos, pues estas son prejuzgadas como inconsistentes, fragmentarias o propias de la charlatanería.

¿Qué conceptos nos permiten superar esta aporía? Uno de ellos es el de símbolo hegemónico propuesto por De Martino (1999), el cual ha sido arrojado al olvido por la literatura antropológica médica. En La tierra del remordimiento, De Martino analiza extraordinariamente el tarantismo y conceptualiza el lugar que ocupa el símbolo de la tarántula como elemento unificador y coordinador de simbolismos heterogéneos de esta manifestación ritual, produciendo un horizonte simbólico emergente ${ }^{23}$. Inspirándonos en él, nos preguntamos si tiene sentido buscar al interior del proceso articulatorio analizado un símil de símbolo hegemónico. Nuestra hipótesis es que el diablo operaría como tal, donde su doble referencia al Saber Pentecostal y al Andino, facilitaría la existencia de articulaciones en distintos niveles del fenómeno, según detallamos a continuación.

1. A nivel de diagnóstico, se constata que en muchos relatos de enfermedades "de maligno", el discurso se refiere más bien a las entidades 
territoriales como causantes de la misma, es decir: son entendidas como "agarraduras" de cerro, de gentil o de viento, pero no como "agarraduras del diablo". Resulta significativo que, a su vez, no exista dicha nomenclatura en el Saber Pentecostal, lo que hipotéticamente evidenciaría la inexistencia de una nosología propia al respecto. Este es uno de los rasgos peculiares de este saber y una clave para entender su capacidad articulatoria, en tanto dicha ausencia supone que ningún problema de salud le es ajeno en principio.

2. En el nivel etiopatogénico también aparece el diablo como elemento coordinador de las lógicas de ambos saberes. Recordemos que según el Saber Andino, la enfermedad se produce por la confluencia del "mal paraje" y la "mala hora". Al interior de la ideología del Saber Pentecostal, el "mal paraje" deja de ser significado como una entidad territorial específica y se transforma en un lugar donde frecuentemente aparece el demonio. Contra la topología de las entidades territoriales, la materia de los lugares se entiende como inanimada. A no ser, una vez más, por la presencia e intervención del maligno.

Más aún, en la significación pentecostal el diablo tiene el carácter polarizado de una entidad malévola, y por esto, cuando se resignifica a la entidad territorial como diabólica, hace que el "mal paraje" devenga siempre malo, pues quien lo anima es el demonio. Esto evidencia la existencia de un sentido muy diverso del concepto de "mal paraje", pues en lo concreto, la "maldad" o "fiereza" con que se les caracterizaba en la concepción aymara, no implicaba un rechazo al establecimiento de relaciones con él. Muy por el contrario, estaba asociada a los cuidados de la reciprocidad, pues de ella dependían los resultados benéficos o perjudiciales de esta relación.

En cuanto a la "mala hora", observamos que ella pierde su especificidad ligada a la existencia de ciertas horas umbrales o al momento donde se producía el quiebre de la reciprocidad. En la concepción pentecostal del mundo, toda hora es potencial momento de tentación, por lo que siempre se arriesga la presencia demoníaca. Lo que ocurre en términos genéricos es que la especificidad de las relaciones que la persona establece con las entidades territoriales es significada como forma de idolatría. Así, pierde sentido el núcleo de la comprensión que posee el yatiri sobre la "agarradura", donde resulta vital preguntarse por la entidad territorial específica ("mal paraje") y por el momento exacto en que se ocasionó la enfermedad ("mala hora").

3. En el nivel terapéutico el diablo también actúa como un elemento coordinador. Si en el Saber Andino la cura consistía en un restablecimiento de la reciprocidad, en la lógica terapéutica pentecostal se reorganiza en torno a la figura del diablo ${ }^{24}$, tanto a través de las prácticas preventivas (p.ej. salmo 91) como de las terapéuticas, las que asumen la forma de una batalla contra él, o de una recuperación del alma de los desvíos pecaminosos.

Como vemos, la doble referencialidad, el diablo como símbolo hegemónico permite articular y coordinar ambos saberes médicos en sus distintos niveles, produciendo un nuevo horizonte simbólico operativo desde el que se enfrentan los problemas de salud.

Siendo consistentes con el potencial heurístico del concepto de símbolo hegemónico, debemos problematizar de qué manera esta articulación de saberes participa de la producción de relaciones de hegemonía/subalternidad ${ }^{25}$. Nuestra evidencia etnográfica muestra que de la coordinación operativa que ejerce este símbolo hegemónico surge una notable hegemonía del Saber Pentecostal sobre el Andino. Si bien esto se debe a muchísimas razones que escapan del foco de nuestra investigación, nos interesa explorar una línea específica de esta manifestación. Mostraremos cómo la producción de estas nuevas formas de eficacia participa del proceso más amplio de deslegitimación del Saber Andino como forma de idolatría ${ }^{26}$.

Mediante las articulaciones de diversos elementos ideológicos, se está produciendo un proceso complejo de apropiación y sustitución de elementos, como también de subordinación en la atribución de la eficacia de estos saberes. Por esta vía se fortalece al Saber Pentecostal y se capitalizan las múltiples experiencias de padecimiento y cura de los sujetos aymaras, a través de un horizonte que simultáneamente toma elementos del Saber Andino y desconoce su contribución al proceso complejo de curación.

$\mathrm{Al}$ interior de la ideología pentecostal, cualquier eficacia atribuida al Saber Andino va a ser explicada como prueba del poder de dios (o del "maligno"), reforzando prácticamente que la explicación y operatividad de este saber debe pasar por la matriz simbólica de la relación entre la persona y dios, y no por la lógica de la reciprocidad con las entidades territoriales. También se muestra que la eficacia de 
los yatirinaja y qullirinaja no puede descansar en la agencia humana, ya que en el Saber Pentecostal toda eficacia es siempre una "eficacia vicaria" que descansa finalmente en el poder de dios. Por último, este saber descalifica masivamente los diversos niveles que componen el Saber Andino, remitiendo siempre a la figura del diablo como símbolo omnipresente.

Pensar en el diablo como un símbolo hegemónico del proceso analizado, permite explicitar que las relaciones articulatorias que se generan entre los saberes médicos participan de las relaciones de hegemonía/subalternidad. Mediante el proceso articulatorio general de constitución de nuevas formas de eficacia, lo que se está jugando en el terreno ideológico es el establecimiento de una hegemonía pentecostal. Esta se muestra relativamente eficaz en la intervención frente al sufrimiento, estableciendo nuevas formas de sentido común desde las cuales representar y actuar en la realidad frente al Proceso Salud/Enfermedad/Atención, e irradiando desde ahí su influjo hacia la interpretación de la realidad total ${ }^{27}$.

\section{Conclusiones}

Estudiar el pluralismo médico desde un enfoque relacional nos ha permitido iluminar fenómenos y dinámicas articulatorias propias del Proceso Salud/ Enfermedad/Atención. Ellas producen nuevas formas de eficacia que exigen ser explicadas por conceptos que, respetando la coexistencia de saberes médicos, permitan entender la operatividad emergente de su articulación. En esta línea, el concepto de símbolo hegemónico nos ha permitido describir y subrayar el rol coordinador y operativo que el símbolo del diablo tiene respecto a otros simbolismos de los saberes en cuestión. Además, hemos apreciado su participación en la producción de la eficacia de las prácticas de atención, y su rol en la producción de relaciones de hegemonía/subalternidad dentro de un proceso histórico más amplio de subordinación que supone la persecución de las "idolatrías" (tanto en su vertiente católica colonial como contemporánea pentecostal).

Consecuentemente, el hecho de que los elementos característicos del Saber Andino estén subordinándose al Saber Pentecostal debe advertirnos sobre la fascinación que pudiera generar la producción de "nuevas formas de eficacia". Estas constituyen formas específicas no solo de expansión y creación de formas terapéuticas, sino también de una trágica historia de intolerancia cristiana ante el Saber Andino y la lógica cultural que la sostiene.

Agradecimientos: A Camiña y su gente. A los evaluadores. Este artículo resulta de la tesis doctoral "La Mala Hora. Articulaciones en el pluralismo médico de agricultores precordilleranos aymaras chilenos", financiada por Becas-Chile de CONICYT.

\section{Referencias Citadas}

Arratia, M. 1992. Problemas conceptuales de la antropología y sus implicancias. Diálogo Andino 11-12:34-45.

Astvaldsson, A. 2004. El flujo de la vida humana. Hueso Húmero 44:89-112.

Bouysse-Cassagne, T. 1978. L'espace aymara. Annales. Économies, Sociétés, Civilisations 33:1057-1080.

Caba, F., S. Alvarado y D. Cáceres 2011. Caracterización epidemiológica y evolución del cáncer de mama en Arica y Parinacota, Chile 1997-2007. Revista de Salud Pública 13:398-409.

Cant, S. y U. Sharma 1999. A New Medical Pluralism? Routledge, London.

Carrasco, X., F. Daiber, P. Rothhammer, D. Huerta, C. Andrade, P. Opazo y F. Aboitiz 2012. Trastorno por déficit de atención e hiperactividad en niños aymara. Revista Médica de Chile 140:1409-16.

Choque, C. 2012. Fortunato Manzano, el Último Yatiri. CONADI, Arica.
Crandon-Malamud, L. 1991. From the Fat of Our Souls. University of California Press, Berkeley.

Cuadro, I. 2008. Acercamiento antropológico del concepto de salud mental en los aymaras del sector Isluga. Revista Cultura y Religión 2:1-12.

De Martino, E. 1999. La Tierra del Remordimiento. Edicions Bellaterra, Barcelona.

De Martino, E. 2004. El Mundo Mágico. Los libros de la araucaria, Buenos Aires.

Descola, P. 2001. Construyendo naturalezas. En Naturaleza y Sociedad: Perspectivas Antropológicas, editado por P. Descola y G. Pálsson, pp. 101-123. Siglo XXI Editores, México, D.F.

Díaz, A. 2012. Procesos de idolatría, discursos nativos y religiosidad en el mundo andino colonial. Taller de Letras 51:53-74.

Estermann, J. 2009. Filosofía Andina. ISEAT, La Paz.

Fernández, G. 2002. Simbolismo Ritual entre Los Aymaras. Tesis para optar al grado de Doctor, Facultad de Geografía e Historia, Universidad Complutense de Madrid, Madrid. 
Fernández, G. 2004. Ajayu, animu, kuraji. En Salud e Interculturalidad en América Latina, editado por G. Fernández Juárez, pp. 279-303. Ediciones Abya-Yala, Quito.

García, P.A. 2005. Aru Pirwa. Tercera edición, Imprenta Graficolor, Iquique.

Gavilán, V., C. Madariaga, N. Morales, M. Parra, A. Arratia y R. Andrade. 2009. Conocimiento y Prácticas en Salud. Oñate Impresores, Iquique.

Gavilán, V., P. Vigueras, M. Parra, C. Madariaga, N. Morales, A. Arratia y R. Andrade 2011. La sociedad y la cultura andina contemporánea. Revista de Indias 71:571-600.

Grebe, M.E. 1983. En torno a los ritos terapéuticos astrales de Isluga. Chungara 10:155-164.

Greenway, C. 1998. Hungry earth and vengeful stars. Social Science \& Medicine 47:993-1004.

Griffiths, N. 1998. La Cruz y la Serpiente. Pontificia Universidad Católica del Perú, Lima.

Guerrero, B. 1995. Medicina Andina y Medicina Pentecostal en los Aymaras del norte grande de Chile. Chungara 27:153-165.

Gundermann, H. 1997. Acerca de cómo los aymara aprendieron el castellano (terminando por olvidar el aymara). Estudios Atacameños 12:89-104.

Gundermann, H. 2009. Sociedades indígenas y conocimiento antropológico. Estudios Atacameños 41:113-164.

Gundermann, H. 2011. Historia moderna de una lengua originaria. RLA. Revista de Lingüística Teórica y Aplicada 49:69-108.

Lagos, L.P., C. Silva, P. Rothhammer, X. Carrasco, E. Llop, F. Aboitiz y F. Rothhammer 2011. Riesgo de déficit atencional/ hiperactividad en escolares Aymara, Rapa-Nui y de Santiago de Chile. Revista Médica de Chile 139:600-605.

Layme, F. 2004. Diccionario Bilingüe Aymara-Castellano. Edición Consejo Educativo Aymara, La Paz.

Leslie, C. 1980. Medical pluralism in world perspective. Social Science \& Medicine 14:191-195.

Makela, M., S. Barton, W.J. Schull, W. Weidman y F. Rothhammer 1978. The multinational Andean genetic and health program, IV. Journal of Chronic Diseases 31:587-603.

Mamani, B. y S. Mendoza 1981. Concepto de los aymaras sobre las enfermedades y sus causas. Boletín del Instituto de Estudios Aymaras 82:22-35.

Mansilla, M.A. 2009. La Cruz y la Esperanza. Editorial Universidad Bolivariana, Santiago.

Martínez, G. 1976. El Sistema de los Uywiris en Isluga. Centro de Investigaciones Andinas, Iquique.

Martínez, G. 1983. Los dioses de los cerros en los Andes. Journal de la Société des Américanistes 69:85-115.

Martínez-Hernáez, A. 2008. Antropología Médica. Anthropos, Barcelona.

Mauss, M. 1971. Sociología y Antropología. Tecnos, Madrid.

Menéndez, E.L. 1994. La enfermedad y la curación. Alteridades 47:71-83.

Menéndez, E.L. 1997. El punto de vista del actor. Relaciones. Estudios de Historia y Sociedad 69:238-270.

Menéndez, E.L. 2003. Modelos de atención de los padecimientos. Ciência \& Saúde Coletiva 8:185-207.
Mitchell, W.P. 1991. Some are more equal than others. Research in Economic Anthropology 13:191-219.

Murra, J.V. 1975. Formaciones Económicas y Políticas del Mundo Andino. Instituto de Estudios Peruanos, Lima.

Orlove, B.S. 1977. Inequality among peasants. En Peasant Livelihood, editado por R. Halperin y J. Dow, pp. 201-226. St. Martin's Press, New York.

Paredes, R. 1920. Mitos, Supersticiones y Supervivencias Populares de Bolivia. ARNO Hermanos-Libreros Editores, La Paz.

Perdiguero-Gil, E. 2006. Una reflexión sobre el pluralismo médico. En Salud e Interculturalidad en América Latina: Antropología de la Salud y Crítica Intercultural, editado por G. Fernández Juárez, pp. 33-49. Ediciones Abya-Yala, Quito.

Piñones Rivera, C. 2015. La Mala Hora. Tesis para optar al grado de Doctor en Antropología, DAFITS, Universitat Rovira i Virgili, Tarragona.

Ramírez Hita, S. 2005. Donde el Viento Llega Cansado. Plural Editores, La Paz.

Rothhammer, P., X. Carrasco, H. Henríquez, C. Andrade, M. Valenzuela, F. Aboitiz y F. Rothhammer 2005. Bajo riesgo de déficit atencional/hiperactividad en niños Aymarás. Chungara Revista de Antropología Chilena 37:145-149.

Severi, C. 2007. Le Principe de la Chimère. Editions rue d'Ulm, Paris.

Stoner, B.P. 1986. Understanding Medical Systems. Medical Anthropology Quarterly 17:44-48.

Taipe, N. 2000. Dos Soles y Lluvia de Fuego en los Andes. Escuela Nacional de Antropología e Historia Perú, Lima.

Tambiah, S.J. 1990. Magic, Science and Religion and the Scope of Rationality. Cambridge University Press, Cambridge.

Taussig, M. 2010. The Devil and Commodity Fetishism in South America. The University of North Carolina Press, Chicago.

Taylor, G. 1980. Supay. Amerindia 5:47-63.

Van den Berg, H. 1989. La Tierra No Da Así Nomás. CEDLA, Amsterdam.

Van Kessel, J. 1983. Ayllu y ritual terapéutico en la medicina andina. Chungara 10:165-178.

Van Kessel, J. 1985. Medicina Andina. CIREN, Iquique.

Van Kessel, J. 2004. Rituales Pastoriles de la Puna. IECTA, Iquique.

Van Kessel, J. y B. Guerrero 1987. Sanidad y Salvación en el Altiplano Chileno. CIREN, Iquique.

Viveiros de Castro, E. 1996. Perspectivismo y multinaturalismo en la américa indígena. En Tierra Adentro: Territorio Indígena y Percepción del Entorno, editado por A. Surrallés y P. García, pp. 37-79. IWGIA, Lima.

Weber, M. 1998. La ética económica de las religiones universales. En Ensayos Sobre Sociología de la Religión I. Taurus, Madrid.

Weiner, A.B. 1992. Inalienable Possessions. University of California Press, London.

Yampara, S. 2005. Comprensión Aymara de la Tierra-Territorio en la cosmovisión andina y su ordenamiento para la/el qamaña. Inti Pacha 1:13-44. 


\section{Notas}

1 Al utilizar este concepto estamos posicionándonos en una perspectiva crítica respecto del Modelo Médico Hegemónico, subrayando el carácter social, cultural, económico-político e histórico del proceso colectivo de enfermar, atender y cuidar la salud. Así, el Proceso Salud/Enfermedad/Atención es entendido como un hecho social estructural, que engloba las formas de padecimientos y las respuestas sociales que se aplican desde y hacia los conjuntos sociales, así como las diversas representaciones y prácticas sociales asociadas. El proceso está socialmente estratificado y participa de los procesos de estratificación social, teniendo una relación expresiva con sus condiciones sociales, culturales, económicas y políticas. Uno de los elementos específicos de la aproximación gramsciana aquí asumida es que este proceso de estratificación es pensado desde el concepto de hegemonía, lo que supone un análisis de las relaciones de hegemonía/subalternidad que se dan entre los distintos actores o saberes implicados.

2 Para una crítica a sus supuestos epistemológicos Cfr. Gundermann (2009) y Arratia (1992).

3 Para análisis del resto de las articulaciones Cfr. Piñones Rivera (2015). En adelante utilizaremos las expresiones "Saber Andino" o "Andino" para referirnos al Saber Médico Andino cuando el contexto nos autorice. Lo mismo ocurrirá con el Saber Médico Pentecostal.

4 La palabra qulliri significa "persona con cualidad de curar" (García 2005). En nuestro campo se utilizaba para referirse a las personas que saben principalmente del uso de hierbas, emplastos o problemas de huesos y nervios. A diferencia, yatiri significa "el que sabe" (Layme 2004) y su conocimiento especializado (si bien no restrictivo), es sobre los llamados "problemas espirituales": susto, agarradura, mal impuesto, etc. (Piñones Rivera 2015). Cfr. también Choque (2012).

5 Si bien el autor principal ha realizado cursos de lengua aymara no la domina en profundidad, por lo que todo el trabajo de campo fue realizado en castellano. Compartiendo que el dominio de la lengua constituye un elemento central en el estudio de los Pueblos Originarios, se ha documentado profusamente el problema de la pérdida de la lengua aymara entre los chilenos (Gundermann 2011; Gundermann 1997), lo que se ve reflejado también en Camiña donde la mayoría de las personas no utiliza la lengua aymara, y solo una fracción la comprende.

6 Un análisis detallado de esta propuesta puede leerse en Menéndez (1997) y Ramírez Hita (2005).

7 En algunos trabajos de Van Kessel y Guerrero (1987) y Guerrero (1995) esto aparece señalado, no obstante, no es suficientemente desarrollado, ni surge del método etnográfico.

8 Al decir que el espacio aymara está conformado por entidades territoriales, buscamos evitar la introducción de una serie de supuestos naturalistas. Articulando las críticas que han esbozado diversos autores, en este artículo no consideraremos a estas entidades como lugares sagrados (Astvaldsson 2004), espíritus de los lugares (Martínez 1983), divinidades (Estermann 2009), no-humanos (Astvaldsson 2004), entidades sobrenaturales (Viveiros de Castro 1996), o proyecciones humanas (De Martino 2004). Al reconocer el estatuto de "entidades" afirmamos su existencia negada por la filosofía materialista moderna (Tambiah 1990; Weber
1998), y al cualificarlas como territoriales ponemos énfasis en que esta existencia debe ser entendida a la luz de la relación que establece el pueblo aymara con su territorio, no pudiendo ser concebida únicamente de manera abstracta e independiente de dicha relación con la dimensión espacial.

9 El problema de una topología andina escapa a los alcances de este artículo. Cfr. los importantes aportes de Van den Berg (1989), Martínez (1976), Greenway (1998), BouysseCassagne (1978), Yampara, Tórrez, y Morales (2005) y Murra (1975).

10 "Ch'allaña: Realizar un acto ritual con licor pidiendo bienestar y prosperidad o que la cosa festejada dure mucho" (Layme 2004:57). Por su parte, "la phawa es una ofrenda grande consistente en fibra de alpaca, llamp' $u$ (grasa de pecho de camélido, un regalo muy apreciado por las divinidades andinas), hojas de coca, confites y sullu (feto de camélido). La phawa no contiene sullu, cuando en la misma oportunidad se sacrifica un animal vivo" (Van Kessel 2004:62).

11 Respecto de la "agarradura" Cfr. Mamani y Mendoza (1981), Paredes (1920), Fernández (2002). Para el caso de Tarapacá, ver Gavilán et al. (2009).

12 Fernández (2004) ha mostrado que el concepto de ánimo no se corresponde con el de espíritu, siendo más acorde el de sombra (ch'iwi). Para una discusión local ver Gavilán et al. (2009).

13 Entendemos a la reciprocidad como una relación de corespondencia entre los actores, cuyo intercambio está sometido a una cierta obligatoriedad, la cual es la fuente de una conciencia ambivalente: se da de buena fe, pero este acto es objeto de una coerción social implícita (Mauss 1971). No desconociendo las críticas que denuncian la función de velamiento de la inequidad (Mitchell 1991; Orlove 1977) y la vehiculización de una "ideología de la norma moral" (Weiner 1992), consideramos que el concepto es útil si se usa de manera acotada. En concreto, consideramos que como forma de co-respondencia, restituye la transitividad característica de la relación entre humanos y entidades territoriales, contribuyendo a pensar en la existencia de una relación propiamente social en la dirección que va desde lo natural a lo social (Descola 2001; Viveiros de Castro 1996), y desnaturalizando así la "reificación de las divinidades andinas" (Griffiths 1998).

14 Este proceso no está únicamente ligado a la iglesia pentecostal, pues fue característico de la "extirpación de idolatrías" (Cfr. Griffiths 1998; Taussig 1980; Taylor 1978). Solo estamos señalando su faceta actual, centrándonos en el foco definido por las articulaciones entre saberes médicos.

15 Los gentiles (castellano) o chullpas (aymara), corresponden a formas previas de humanidad. Son caracterizados como seres pequeños que vivían de una agricultura regida solo por el ciclo lunar y cuya forma de vida fue destruida con la salida del sol, quedando como vestigios sus pequeñas construcciones y antiguos corrales, con sus valiosos objetos y huesos en miniatura (Taipe 2000).

16 Pirqar es una forma de construcción consistente en apilar piedras. Es usado en corrales, terraplenes, andenes, etc.

17 La turca es el tratamiento consistente en un intercambio ritual sacrificial de un animal a cambio del ánimo de la persona. 
18 "Probando" y "en plena" son categorías que señalan el avance del compromiso en el camino pentecostal.

19 Tipo de tejido andino con usos productivos, estéticos y rituales.

20 Se refiere a la "imposición de manos", una de las prácticas rituales de sanación distintivas del pentecostalismo.

21 Sobre la importancia terapéutica de la imposición de manos, ver Mansilla (2009). Sobre la relevancia de la matriz personadios para la comprensión del Saber Pentecostal ver Piñones Rivera (2015).

22 Para una visión divergente sobre la eficacia simbólica véase Severi (2007).

23 De su propuesta nos interesa destacar la dimensión de articulación fragmentaria que posee este simbolismo. Desatenderemos la supuesta función de integración totalizante, pues no se ajusta a nuestra experiencia de campo ni perspectiva de análisis.

24 Aquí debemos enfatizar que nos referimos a las enfermedades características del Saber Andino, pues en aquellas que obtienen su nomenclatura y sentido del Saber Biomédico, la lógica terapéutica se mimetiza con las de ese saber. Así, varias enfermedades de cuño biomédico eran tratadas desde una biomedicina traspuesta al plano espiritual.

25 Este es uno de los elementos del concepto de Símbolo Hegemónico que lo diferencia del concepto de Símbolo Dominante propuesto por Turner, en el cual halla clara influencia. En concreto, esta dimensión evidencia su vínculo con la aproximación gramsciana, haciendo confluir tanto la riqueza hermenéutica como la determinación económicopolítica de los fenómenos simbólicos (Martínez-Hernáez 2008).

26 Estrategia que tiene cierta continuidad con el proceso de extirpación de idolatrías colonial (Díaz 2012).

27 La dinámica de subalternización que aquí analizamos se sitúa dentro de la historia de subordinación de la cultura andina bajo el concepto de idolatría (Taussig 2010; Griffiths 1998; Taylor 1980). Pero además, está relacionado con el proceso paralelo de articulaciones entre el Saber Biomédico y el Andino, el cual es conducido por el Estado chileno a través de su política multiculturalista neoliberal de Salud Intercultural (Piñones Rivera 2015). 
